\title{
El Mobbing y los Síntomas de Estrés en Docentes Universitarios del Sector Público
}

\author{
MOBBING AND SYMPTOMS OF STRESS IN PUBLIC SECTOR UNIVERSITY FACULTY
}

\author{
Lara Sotomayor Juan Eduardo', Manuel Pando Moreno ${ }^{2}$ \\ 1. Instituto de Investigación y Postgrado - Facultad de Psicología, Universidad Central del Ecuador. \\ 2. Instituto de Investigación en Salud Ocupacional - Universidad de Guadalajara - México.
}

\begin{abstract}
RESUMEN
Este artículo trata sobre la presencia de los factores psicosociales en el trabajo. El objetivo del estudio fue identificar la presencia e intensidad de la violencia psicológica, el acoso laboral, los factores sociodemográficos laborales como: género, edad, estado civil, tiempo de dedicación y nivel de formación; y, establecer la relación con los niveles de estrés en docentes universitarios del sector público en Ecuador. Se avaluó a 187 docentes principales de la Universidad Central del Ecuador. Se aplicó el Inventario de Violencia y Acoso Psicológico en el Trabajo (IVAPT-PAND0), el Inventario de Síntomas de Estrés (ISE) y la encuesta de Factores Socio-demográficos Laborales (ESOLA). Los resultados: un $87,2 \%$ de los investigados (163 docentes) manifiesta presencia de violencia psicológica; el 91,9\% (172 docentes) indica que la intensidad de la violencia psicológica es baja y nula; sobre el Acoso Psicológico (mobbing) 183 docentes $(97,8 \%)$ sostienen que es nulo o bajo; 4 docentes $(2,2 \%)$, que es medio y alto; sobre los niveles de estrés, 185 docentes $(98,9 \%)$ indicaron que el nivel es bajo, 2 docentes $(1,1 \%)$, que es medio; los síntomas más frecuentes son: dolores de cuello o espalda, explosiones de coraje, fatiga y gastritis. No existe asociación estadísticamente significativa entre las variables investigadas.
\end{abstract}

(Sotomayor L, Pando M, 2014. El Mobbing y los Síntomas de Estrés en Docentes Universitarios del Sector Público. Cienc Trab. Ene-Abr; 16 [49]: 43-48).

Palabras Claves: MOBBING, VIOLENCIA PSICOLÓGICA, ESTRÉS, SÍNTOMAS DE ESTRÉS, SALUD LABORAL.

\section{ABSTRACT}

This article is about the presence of psychosocial factors at work. The aim of the study was to identify the presence and intensity of psychological violence, bullying, socio -demographic factors such as: gender, age, marital status, and time commitment, level of training and establish the relationship with stress levels University Teachers in the public sector in Ecuador. 187 principal teachers of the Central University of Ecuador were evaluated. Inventory Violence and Bullying at Work (IVAPT - Pando), the Stress Symptom Inventory (ISE) and the Survey of Socio-Demographic Factors Labor (ESOLA) were applied. Results: $87.2 \%$ of the investigated (163 teachers) manifest presence of psychological violence, 91.9\% (172 teachers) indicate that the intensity of psychological violence is low and nil, about Bullying (mobbing) 183 teachers (97.8\%) say it is zero or low, 4 teachers $(2.2 \%)$ is medium and high, about stress levels over 185 teachers $(98.9 \%)$ indicated that the level is low, 2 teachers $(1.1 \%)$ is medium, the most common symptoms are: sore neck or back, outbursts of anger, fatigue and gastritis. No statistically significant association exists between the variables investigated.

Key words: MOBBING AND PSYCHOLOGICAL VIOLENCE, STRESS, SYMPTOMS OF STRESS, OCCUPATIONAL HEALTH.

\section{INTRODUCCION}

Según Herranz-Bellido y Reig-Ferrer ${ }^{1}$ los(as) trabajadores(as) sociales - policias, maestros, profesores, enfermeras y otras profesiones similares- están sometidos a altas exigencias emocionales. Este interés relacionado con la salud en el trabajo no se había dado con la misma intensidad en el caso específico del sector de los servicios, y menos aún en el campo correspondiente a la educación superior; en los últimos años esta tendencia se ha

Correspondencia / Correspondence:

Juan Eduardo Lara Sotomayor

Universidad Central del Ecuador

e-mail: juanelarasmdth@hotmail.com, manolop777@yahoo.com.mx

Recibido: 28 de Diciembre de 2013 / Aceptado: 07 de Marzo de 2014 venido modificando, con aportaciones tanto teóricas como con estudios empíricos en el mundo del trabajo, que abordan fenómenos que se perfilan como emergentes, tales como el mobbing y el estrés laboral.

El fenómeno del mobbing, entendido básicamente como una forma de abuso psicológico que ocurre en el lugar de trabajo, permaneció invisible durante mucho tiempo., ${ }^{2,3}$ Sin embargo, desde la década del 80 el estudio del mobbing tomó auge y en la década siguiente es tal el interés sobre el tema que algunos han llegado a denominarlo «el tópico de la investigación de los años 90" en el campo de la psicología de las organizaciones. ${ }^{4}$

Leymann ${ }^{5}$ indica que con el mobbing se pretende la destrucción psicológica de la víctima por medio de actuaciones más o menos hostiles que consideradas de forma aislada podrian aparecer anodinas, pero cuya repetición produce efectos muy perjudiciales. El mobbing requiere que se reúnan cuatro características, entre la que se destaca el propósito sobre todas las demás, dado que el propósito es una característica indispensable para la diferenciación entre el mobbing y la violencia psicológica generalizada en 
un ambiente laboral; estas características son: 1) existe como una serie de actos violentos, generalmente en lo moral y psicológico, y muy extrañamente de manera física; 2) debe ocurrir con repetitividad o continuidad; 3) se aplica con el propósito de perjudicar la integridad de la otra persona, tratando de obtener su salida de la organización; y, 4) que los comportamientos psicológicamente agresivos sean más o menos verificables. ${ }^{6}$

González de Rivera ${ }^{7}$ ha propuesto la existencia de una nueva patología y la ha denominado "Síndrome de Acoso", donde el factor estresante principal son las relaciones interpersonales negativas con los demás miembros del grupo laboral, que no son ni siquiera conflictivas sino, peor aun, decididamente persecutorias. Difiere en eso de las otras formas de estrés, en las que el factor estresante principal es la propia naturaleza del trabajo, que puede carecer de experiencias positivas, con acumulo de aspectos negativos, o ser traumático, peligroso o ansiógeno en sí mismo, o producir tensión por una excesiva carga de responsabilidad sobre otras personas. Peralta ${ }^{8}$ sostiene que El acoso laboral, mobbing, puede generar síntomas asociados al estrés postraumático, que incapacitan y lesionan psicológicamente a las personas de manera grave. Riquelme $^{9}$ indica que los efectos del Mobbing, o Acoso Psicológico, para las víctimas pueden ser devastadores desde el punto de vista clínico, dando origen a trastornos de ansiedad generalizada, cuadros de estrés, estados depresivos severos pudiendo llegar en grados extremos hasta el suicidio. Los sintomas más persistentes, incluso en personas que parecen haber superado definitivamente el Síndrome de Acoso, son: a) Recuerdos obsesivos, b) Presión Focalizada del Pensamiento y c) temor al lugar de trabajo y a todo lo que le pueda recordar los acontecimientos estresantes y los conflictos que en él tuvieron lugar. Otro estudio realizado por González de Rivera ${ }^{10}$ sostiene que -entre las repercusiones clínicas del acoso psicológico y los síndromes crónicos del estrésel factor estresante principal lo constituyen las relaciones interpersonales negativas con los demás miembros del grupo laboral, que no son ni siquiera conflictivas sino, mucho peor aun, deliberadamente persecutorias.

Bustos $^{11}$ indica que “...los trabajadores se dieron cuenta solo mucho después de que habían sido víctimas de la violencia en el trabajo, reconocieron que estaban siendo tratados mal, pero no en el momento oportuno, hasta que esta situación se convirtió en insoportable, más aún cuando debían permanecer en el mismo sitio de trabajo que el abusador".

Los estudios realizados en los últimos 10 años sobre mobbing y estrés en el espacio laboral -con su sintomatología- indican que la presencia de la violencia psicológica en el trabajo se encuentra en niveles importantes; asi tenemos que, en Colombia, el Ministerio de Trabajo en el año 2004 llevó a cabo un estudio en que se reportó una prevalencia de acoso psicológico del 19,8\%. Los sectores económicos más vulnerables fueron el de transporte y telecomunicaciones con una prevalencia de $(25,1 \%)$, el de salud y educación con $(19,7 \%)$ y el sector financiero con un $(14,6 \%) .{ }^{12}$ Herranza-Bellido, Reig-Ferrer y Cabrero Garcia ${ }^{13}$, realizan un estudio en donde analizan la presencia de mobbing en el profesorado universitario; los resultados indican que casi el 22,6\% de los profesores se sintió victima de mobbing. Los autores sostienen no haber encontrado diferencias estadísticamente significativas en mobbing debidas a la edad, género o categoría académica de los profesores.

En México, Aldrete, Pando, Aranda y Torres ${ }^{14}$ realizan un estudio en donde buscan determinar la presencia del fenómeno entre mujeres docentes de un centro universitario. En este estudio participaron 56 docentes a las que se aplicó el Leyman Inventory of Psycho-Terror (LIPT-60) y la Escala de Evaluación de Apoyo y Contactos Sociales; el estudio hace referencia a la presencia de al menos una estrategia de acoso como mobbing; el 83,6\% de las mujeres se calificaron como víctimas. En referencia al grupo de mujeres participantes, las variables de edad y estado civil no mostraron asociación significativa con ninguna de las diferentes mediciones del mobbing, conforme al LIPT-60.

Justicia ${ }^{15}$ presenta un estudio descriptivo realizado con empleados universitarios de dos universidades andaluzas pertenecientes al sector del profesorado y del personal de administración y servicios; los resultados indican que el 22,3\% de ellos ha sufrido acoso por lo menos una vez durante su experiencia laboral. El porcentaje desciende hasta el 9,31\% al introducir criterios de valoración más restrictivos tales como la frecuencia y la duración. Es importante indicar que el acoso afecta por igual a los grupos analizados y no existen diferencias significativas ante la presencia de este factor; sin embargo, en referencia al sexo existen diferencias estadísticamente significativas, observando que la tasa de victimización en los hombres es de 6,85\%, mientras en las mujeres es el $12,77 \%$. En cuanto a la direccionalidad del acoso, se indica que el acoso descendente afecta a un 76,5\% de las víctimas; el acoso horizontal afecta a un 56,9\% y el acoso descendente afecta al $39,2 \%$ de las víctimas.

Raya, Herruzo y Pino ${ }^{16}$ realizaron la medición de los niveles de acoso psicológico en una población de 220 profesores (93 hombres y 127 mujeres) de los centros de educación de la Provincia de Córdova, considerando los factores socio-demográficos y el estudio de las conductas de acoso más frecuentes que se usan. Los resultados indican que son 4 de las 60 conductas de acoso psicológico; los factores socio-demográficos no guardan ninguna relación directa con la presencia de mobbing. Sobre las conductas de acoso psicológico, la comunicación y la reputación personal son las conductas más indicadas de haber sido violentadas.

Pando $^{17}$, en el estudio sobre Factores psicosociales y presencia de mobbing en profesores universitarios, con la aplicación IVAPTPANDO (Inventario de Violencia y Acoso Psicológico en el Trabajo), detecta 9 posibles casos de Acoso Psicológico, 2 en nivel alto y 7 en nivel medio. Por otro lado hasta un $82 \%$ del personal afirma haber recibido alguna forma de violencia psicológica en el trabajo. Desde hace tiempo se sostiene que el estrés es un factor que acompaña el trabajo docente; esto se fundamenta en una revisión de 71 investigaciones realizada (Hiebert y Farber ${ }^{18}$,por ejemplo, encontraron evidencias para considerar a la docencia como una profesión estresante).

Matud $^{19}$ evalúa el estrés laboral y salud en el profesorado; la muestra estuvo conformada por 223 profesionales de enseñanza (155 mujeres y 68 hombres). Los resultados indican que la insatisfacción con el rol y los cambios negativos e incontrolables en la presión laboral, correlacionan con sintomatología de tipo somático, depresiva, de ansiedad e insomnio; y de forma negativa con apoyo social, aunque los coeficientes son bajos. León y Avargues ${ }^{20}$ realizan el estudio "Evaluación del estrés laboral en personal universitario" mediante la aplicaciòn de 315 encuestas: los resultados indican que le prevalencia del estrés laboral fue alta (13\%). La falta de recursos, la sobrecarga de trabajo, el conflicto y la ambigüedad de rol fueron determinantes el momento de analizar la información obtenida. 
Cito el trabajo desarrollado por Cruz, Ovalle y Pando ${ }^{21}$, cuyo objetivo central fue identificar la incidencia de la sintomatología de estrés y mobbing, aportando al estudio de esta problemática con insumos importante como: "Con respecto a la violencia psicológica encontramos 47 académicos -que representan al 88,7\% de la muestra de estudio- que sufren o han sufrido este tipo de situaciones. Del total de los casos, el 61,7\% sostiene que la frecuencia ha sido alta y el 19,2\% media y el mismo porcentaje sostiene que la frecuencia ha sido baja. De acuerdo a la intensidad, el 95,7\% de ellos manifiestan que la violencia recibida es de baja intensidad y únicamente el 4,3\% de los académicos manifiesta que ha sido alta.

“....De los datos más significativos se encuentra que, del total de los casos, el $63,6 \%$ son hombres y el $36,4 \%$ son mujeres, observándose que los hombres son más proclives a experimentar síntomas de estrés; con relación a la edad, los casos se concentran en sujetos que están viviendo su tercera o cuarta edad. Los sintomas que tienen mayor incidencia en los académicos son en orden de importancia: dolores de cabeza, dificultades para quedarse dormidos o despertarse durante la noche, irritabilidad o enfurecimiento, dolores abdominales, nerviosismo o ansiedad y fatiga o debilidad".

Pozos, Horta, Delgadillo y Aguilera ${ }^{22}$ abordan la problemática de la docencia y estrés como riesgo para la salud en odontólogos; para este estudio se definió una población de 90 docentes odontólogos de ambos sexos. Los niveles de estrés crónico que se encontraron fueron: 11 odontólogos $(12,2 \%)$ de la población total presentaron un nivel alto, $62(68,8 \%)$ presentaron un nivel medio y $17(18,9 \%)$ presentaron un nivel bajo. Las autoras sostienen que estos resultados concuerdan con otros estudios realizados donde se hace referencia a que, si bien el estrés afecta a ambos sexos, el impacto en cada uno es diferente. Con relación a la edad se sabe que a mayor edad se va reduciendo la capacidad de su sistema inmune, mostrando significativamente más estrés y si a ello le agregamos alteraciones en la estabilidad emocional por el estrés vivido a lo largo de varios años, las consecuencias pueden ser lamentables.

Necsoi ${ }^{23}$ hace referencia a la relación del estrés generado por el trabajo y a la satisfacción del Docente Universitario; en el estudio participaron 70 docentes universitarios, encontrándose que existe una relación negativa entre la satisfacción en el trabajo y la presencia del estrés laboral; las mujeres presentaron altos niveles de ansiedad y depresión y bajo nivel de satisfacción.

El objetivo de este estudio es identificar la presencia e intensidad de las variables sujetas a estudio, así como la relación con ciertos factores socio-demográficos laborales de los docentes universitarios del sector público y su incidencia en la salud del docente universitario a través de la presencia de ciertos sintomas propios del estrés.

\section{MATERIAL Y MÉTODO}

Este estudio es de tipo relacional, con corte transversal, aplicado a una muestra aleatoria simple conformada por 187 docentes de las seis facultades que forman parte del área de las ciencias de la vida, con nombramiento definitivo y al menos 1 año de antigüedad en la Institución, con tiempo de dedicación completo, medio tiempo y tiempo parcial. La población total en las seis facultades sujetas a estudio es de 595 docentes; para la definición de la muestra se asumió una prevalencia esperada de 50\%, un margen de error aceptable del 0,05 y un nivel de confidencialidad del 95\%, quedando un total de 187 sujetos. Para la selección de sujetos se distribuyó proporcionalmente entre las 6 facultades existentes y se eligieron al azar.

\section{Los instrumentos aplicados a la población sujeto del presente estudio fueron:}

El IVAPT-PANDO (Inventario de Violencia y Acoso Psicológico en el Trabajo), creado por Manuel Pando Moreno, que mide la frecuencia e intensidad de la violencia psicológica y la presencia de Acoso Psicológico-Moobing. Este instrumento consta de 22 items, de doble respuesta en escala likert, que indica si la persona sufre violencia en el trabajo y si la violencia puede ser calificada como acoso psicológico (Mobbing). Fue validado con un alfa de Cronbach de 0.911 y un 36,5\% de varianza explicada para el primer factor.

Dentro de esta primera referencia se encuentra la efectuada por una serie de investigadores, quienes con Pando ${ }^{24}$ la realizaron a través del estudio "Validez y Confiabilidad del Inventario de Violencia y Acoso Psicológico en el Trabajo (IVAPT-PANDO) en la población mexicana. Dicha muestra estuvo constituida por 307 trabajadores de catorce estados de la República Mexicana y veinticuatro giros empresariales diferentes. Tal y como lo señala dicho estudio, los resultados muestran consistencia interna de cuatro factores, con validez relevante y alta confiabilidad. ${ }^{25}$

Como lo señala Pando ${ }^{24}$, "estudios previos realizados (Pando, Aranda, Aldrete y Reynaga) con instrumentos como el LIPT-60 muestran una prevalencia excesiva $(79,7 \%)$ como para poder calificar realmente como acoso laboral, lo que ha obligado a reconsiderar la utilidad de tal instrumento". De igual forma señala "consideraba necesario revisar los constructos de instrumentos ya existentes como el mencionado LIPT-60 y el inventario CISNEROS, pues se halló que no satisfacen los criterios para determinar confiablemente el acoso laboral".

Para determinar el acoso laboral se requiere de tres elementos mínimos: la presencia de comportamientos violentos de una o varias personas sobre otra(s); que estos comportamientos se presenten de manera continua durante cierto tiempo, y que exista la intencionalidad de destrucción psicológica y obtener de esta forma la salida de la organización, o dañar deliberadamente a la persona. ${ }^{25}$

En el estudio "Aplicación y evaluación psicométrica del test IVAPT-PANDO” realizado por Macías ${ }^{26}$ en dos centros de atención primaria, se buscaba evaluar las propiedades psicométricas del test IVAPT-PANDO en una muestra piloto constituida por 48 trabajadores de dos centros de salud especializados en atención primaria ubicados en la región metropolitana de Santiago de Chile. Los resultados arrojan una alta confiabilidad total y una adecuada capacidad discriminativa de los items. ${ }^{25}$

El IVAPT-PANDO en su aplicación en Bolivia y Ecuador ${ }^{27}$ generó los siguientes insumos. Se trató de un estudio transversal, descriptivo, con una muestra aleatoria no representativa de la población económicamente activa en Bolivia y Ecuador. Participaron 620 sujetos: 311 bolivianos y 309 ecuatorianos pertenecientes al sector de la economía formal. La estructura factorial del inventario se evaluó mediante la opción del método de componentes principales y rotación varimax para maximizar la independencia entre los factores. En la iteración inicial se obtuvieron seis factores que explican el 62,8\% de la varianza. Los valores eigen se encuentran por encima de 0,41 ; de los resultados se obtuvo un coeficiente de fiabilidad ${ }_{-}=0,89$ alpha de Cronbach. 
El CUESTIONARIO-ISE (Inventario de Síntomas de Estrés), elaborado y validado por Lipp y Guevara, a su vez traducido y adaptado para México por Domínguez, Méndez y Meza $^{28}$ (1994), mide la intensidad del estrés laboral. El ISE es un instrumento de aplicación individual y colectiva de fácil comprensión y rápida calificación, con un alfa de Cronbach de 0.94, que nos indica un grado aceptable de confiabilidad. ${ }^{28}$

Contiene una lista de 42 sintomas psicofisiológicos característicos del estrés crónico, los cuales se califican en una escala tipo Likert de 6 opciones que van desde nunca a siempre. Este instrumento, construido para la investigación del estrés, incluye un modelo de estudio de los factores psicosociales desencadenantes de la respuesta de estrés, los mediadores cognoscitivos y la investigación y registro de respuestas psicofisiológicas que dan cuenta de la activación del sistema nervioso. Considerando que la presencia de síntomas psicofisiológicos puede determinar la presencia de un estrés alto, medio y bajo, sin ser relativo a lo que se presente en un grupo determinado. La forma de calificación del ISE en esta adaptación asigna un valor de 0 a 5 a cada una de las opciones de respuesta. Domínguez y cols. ${ }^{29}$ validaron el ISE en población mexicana y encontraron un alfa de Cronbach de 0,94; en esta investigación el alfa fue de 0,893.30

El instrumento Encuesta de Datos Socio-demográficos Laborales (ESOLA) fue elaborado considerando 2 condiciones establecidas: condiciones socio-demográficas y condiciones laborales de los investigados.

Los análisis estadísticos desarrollados son tanto descriptivos como de asociación. Los primeros tuvieron la intención de conocer a la población de estudio y obtener las prevalencias respectivas. Dentro del análisis de tipo descriptivo se obtuvieron frecuencias, porcentajes y promedios, mientras que para el análisis de asociación se utilizó la Chi cuadrada (con o sin corrección de Yates), siendo significativa una $\mathrm{p}<0,05$, y estableciendo el OR (odd ratio), considerando presencia de riesgo si el OR es mayor que 1 ; la unidad no se encuentra en el rango de confianza y la p es $<0,05$. El análisis de la información se realizó por medio del programa de cómputo estadístico EXCEL Y SPSS V21, a través de los cuales se analizaron los datos socio-demográficos laborales y se cruzaron los resultados de la presencia e intensidad de la violencia psicológica, la presencia de mobbing y las manifestaciones psicosomáticas generadas por la presencia del estrés.

\section{RESULTADOS}

Los instrumentos indicados se aplicaron a 187 profesores, la edad promedio fue de 52,01 años, con un rango que varió de 26 a 76 años de edad, con una mediana de 52 años y una moda de 60 años; 136 docentes fueron hombres $(72,7 \%)$ y 51 docentes fueron mujeres (27,3\%); en referencia al estado civil, 139 docentes (74,3\%) son casados, $21(11,2 \%)$ son solteros, $22(11,8 \%)$ son divorciados, $2(1,1 \%)$ son viudos y $3(1,6 \%)$ tienen unión libre; sobre el nivel de formación, el 95,6\% tienen cuarto nivel; sobre el tiempo de dedicación, 107 docentes -que representan el 57,2\% - trabajan a tiempo completo, 48 -que representan el 25,7\%- trabajan a medio tiempo y 32 docentes -que representan el 17,1\%- trabajan a tiempo parcial. Es importante indicar que, sobre la antigüedad, 138 docentes -que representan el 73,8\%- indican tener 5 años o más en la Institución. Sobre violencia psicológica en el trabajo, un 87,2\% de los investigados (163 docentes) indica que sí existe, mientras que 24 docentes $(12,8 \%)$ sostienen que no existe este factor. La media de este factor es de 6,58 que, en la escala de presencia de violencia psicológica, se ubica en el nivel alto. En cuanto a la intensidad de la violencia, 172 docentes $(91,9 \%)$ indican que es baja y nula, 14 $(7,6 \%)$ indican que es media y 1 docente $(0,5 \%)$ indica que es alta; la media de este factor es de 10,3 que, en la escala de puntajes sobre intensidad, indica un nivel bajo. Sobre el Acoso Psicológico (mobbing) 183 docentes (97,8\%) indican que es nulo o baja, mientras 4 docentes $(2,2 \%)$ indican que es medio y alto.

\section{Cuadro 1.}

Presencia-Intensidad de la Violencia Psicológica y Acoso Laboral en Docentes Universitarios de Quito-2013.

\begin{tabular}{|c|c|c|c|c|c|c|c|c|}
\hline & \multicolumn{3}{|c|}{ Presencia } & \multicolumn{3}{|c|}{ Intensidad } & \multicolumn{2}{|c|}{ Mobbing } \\
\hline & Bajo & $\begin{array}{l}\text { Medio } \\
\text { Alto }\end{array}$ & $\begin{array}{l}\text { Nula } \\
\text { Baja }\end{array}$ & M & Alta & Bajo & & Alto \\
\hline Homb & $\begin{array}{c}12 \\
(8,8 \%)\end{array}$ & $\begin{array}{c}124 \\
(91,2 \%)\end{array}$ & $\begin{array}{c}126 \\
(92,6 \%)\end{array}$ & $\begin{array}{c}10 \\
(7,4 \%)\end{array}$ & $\begin{array}{c}0 \\
(0 \%)\end{array}$ & $\begin{array}{c}134 \\
(98,5 \%)\end{array}$ & $\begin{array}{c}1 \\
(0,7 \%)\end{array}$ & $\begin{array}{c}1 \\
(0,7 \%)\end{array}$ \\
\hline Muje & $\begin{array}{c}12 \\
(23,5 \%\end{array}$ & $\begin{array}{c}39 \\
(76.4 \%)\end{array}$ & $\begin{array}{c}46 \\
(90,2 \%)\end{array}$ & $\begin{array}{c}4 \\
(7,8 \%)\end{array}$ & $\begin{array}{c}1 \\
(2 \%)\end{array}$ & $\begin{array}{c}49 \\
(96,1 \%)\end{array}$ & $\begin{array}{c}1 \\
(2 \%)\end{array}$ & $\begin{array}{c}1 \\
(2 \%)\end{array}$ \\
\hline
\end{tabular}

Los niveles de estrés que presentó la población investigada fueron: 185 docentes $(98,9 \%)$ indicaron que el nivel es bajo, mientras 2 docentes $(1,1 \%)$ indicaron que el nivel es medio; los síntomas más frecuentes son:

Cuadro 2.

Síntomas frecuentes de estrés en los Docentes Universitarios de Quito - 2013.

$\begin{array}{ll} & \text { Síntomas del estrés crónico } \\ \text { Dolores de cuello o espalda } & 1,28 \\ \text { Explosión de furia } & 0,88 \\ \text { Fatiga } & 0,87 \\ \text { Gastritis } & 0,85 \\ \text { Insomnio } & 0,84 \\ \text { Dolor de cabeza } & 0,81\end{array}$

De los datos más significativos en el análisis de las variables, encontramos que 161 docentes $(86,1 \%)$-que han sido sujetos de violencia psicológica- manifiestan un bajo nivel de estrés; 171 docentes $(91,4 \%)$-que indican que la intensidad de la violencia psicológica es baja/nula - también indican bajo nivel de estrés, en tanto que 182 docentes (97,3\%) indican la no presencia de mobbing, por lo tanto, el nivel de estrés también se ubica en un nivel bajo.

En referencia a las variables sociodemográficas y su relación con las variables de violencia psicológica, mobbing y niveles de estrés, los datos que se obtienen indican que no existe una asociación estadística significativa, según el Cuadro 4.

\section{DISCUSIÓN}

La presencia de los factores psicosociales en el trabajo en la actualidad ha generado una especial atención por parte de los profesionales encargados de mantener la Salud de los trabajadores, quienes, en base a los múltiples estudios realizados sobre todo en violencia y acoso psicológico en el trabajo -como en la presencia de estrés y su sintomatología más frecuente- han logrado generar una real conciencia en los diferentes actores sobre los efectos dañinos que producen en los trabajadores. Los resultados de la investigación arrojan una población con características socio-demográficas laborales comunes a pobla- 
Cuadro 3.

Análisis de variables.

\begin{tabular}{|c|c|c|c|c|c|c|c|}
\hline \multirow{3}{*}{$\begin{array}{l}\text { Presencia } \\
\text { de Violencia } \\
\text { Psicológica }\end{array}$} & \multicolumn{4}{|c|}{$\begin{array}{l}\text { Niveles de Estrés } \\
\text { Bajo \% Nivel N }\end{array}$} & $\%$ & \multicolumn{2}{|c|}{$\begin{array}{c}\text { Investigados } \\
\text { Casos / Válidos Total }\end{array}$} \\
\hline & Alta & 161 & $86,1 \%$ & 2 & $1,1 \%$ & 187 & $87,2 \%$ \\
\hline & Nula & 24 & 12,8 & . & $0,0 \%$ & & $12,8 \%$ \\
\hline \multirow{2}{*}{$\begin{array}{l}\text { Intensidad } \\
\text { de Violencia } \\
\text { Psicológica }\end{array}$} & Alt & 14 & & 1 & $0,5 \%$ & 187 & $8,0 \%$ \\
\hline & Nula & 171 & $91,4 \%$ & 1 & $0,5 \%$ & & $92,0 \%$ \\
\hline $\begin{array}{l}\text { Mobbing / } \\
\text { Acoso Laboral }\end{array}$ & $\begin{array}{l}\text { Alta - Media } \\
\text { Nula }\end{array}$ & $\begin{array}{c}3 \\
182\end{array}$ & $\begin{array}{l}1,6 \% \\
97,3 \%\end{array}$ & $\begin{array}{l}1 \\
1\end{array}$ & $\begin{array}{l}0,5 \% \\
0,5 \%\end{array}$ & 187 & $\begin{array}{l}2,1 \% \\
97,9 \%\end{array}$ \\
\hline
\end{tabular}

Cuadro 4.

Variables sociodemográficas y su relación con las variables de violencia psicológica, mobbing y niveles de estrés.

\begin{tabular}{|c|c|c|c|c|c|c|c|c|}
\hline \multirow[b]{2}{*}{ EDAD } & \multicolumn{2}{|c|}{ Presencia VP } & \multicolumn{2}{|c|}{ Intensidad VP } & \multicolumn{2}{|c|}{ Mobbing } & \multicolumn{2}{|c|}{ Síntomas de Estrés } \\
\hline & $\begin{array}{l}\text { Alta-- } \\
\text { Media }\end{array}$ & Nula & $\begin{array}{l}\text { Alta - } \\
\text { Media }\end{array}$ & $\begin{array}{l}\text { Baja - } \\
\text { Nula }\end{array}$ & $\begin{array}{l}\text { Alta-- } \\
\text { Media }\end{array}$ & $\begin{array}{l}\text { Nula } \\
\text { /Baja }\end{array}$ & Bajo & Medio \\
\hline $26-45$ & 37 & 11 & 1 & 47 & 1 & 47 & 48 & 0 \\
\hline $46-76$ & 126 & 13 & 14 & 125 & 3 & 136 & 137 & 2 \\
\hline \multicolumn{3}{|c|}{ Chi-cuadrado de Pearson: 0,015 } & \multicolumn{2}{|r|}{0,079} & \multicolumn{2}{|r|}{0,975} & \multicolumn{2}{|r|}{0,403} \\
\hline $\begin{array}{l}\text { ESTADO } \\
\text { CIVIL }\end{array}$ & $\begin{array}{l}\text { Prese } \\
\text { Alta- } \\
\text { Media }\end{array}$ & $\begin{array}{l}\text { ia VP } \\
\text { Nula }\end{array}$ & $\begin{array}{l}\text { Intens } \\
\text { Alta - } \\
\text { Media }\end{array}$ & $\begin{array}{l}\text { Iad VP } \\
\text { Baja - } \\
\text { Nula }\end{array}$ & $\begin{array}{l}\text { Mob } \\
\text { Alta - } \\
\text { Media }\end{array}$ & $\begin{array}{l}\text { bing } \\
\text { Nula } \\
\text { / Baja }\end{array}$ & $\begin{array}{r}\text { Sintomas } \\
\text { Bajo }\end{array}$ & $\begin{array}{l}\text { de Estrés } \\
\text { Medio }\end{array}$ \\
\hline Casado & 121 & 18 & 8 & 131 & 2 & 137 & 138 & 1 \\
\hline S-V-S-UL & 42 & 6 & 7 & 41 & 2 & 46 & 47 & 1 \\
\hline
\end{tabular}

Chi-cuadrado de Pearson: $0,936 \quad 0,052 \quad 0,260 \quad 0,428$

\begin{tabular}{|c|c|c|c|c|c|c|c|c|}
\hline TIEMPO & Prese & sin VP & Inte & VP & Mot & na & omas & Estrés \\
\hline $\begin{array}{l}\text { DE } \\
\text { DEDICACIÓN }\end{array}$ & $\begin{array}{l}\text { Alta - } \\
\text { Media }\end{array}$ & & $\begin{array}{l}\text { Alta- } \\
\text { Media }\end{array}$ & $\begin{array}{l}\text { Baja - } \\
\text { Nula }\end{array}$ & $\begin{array}{l}\text { Alta-- } \\
\text { Media }\end{array}$ & $\begin{array}{l}\text { Nula } \\
\text { / Baja }\end{array}$ & Roir & \\
\hline Completo & 100 & 7 & 8 & 99 & 2 & 105 & 106 & 1 \\
\hline MT-Parcial & 126 & 13 & 7 & 73 & 2 & 78 & 79 & 1 \\
\hline
\end{tabular}

Chi-cuadrado de Pearson: 0,003 $\quad 0,751 \quad 0,768 \quad 0,836$

\begin{tabular}{|c|c|c|c|c|c|c|c|c|}
\hline \multirow[b]{2}{*}{$\begin{array}{l}\text { FORMA- } \\
\text { CIÓN }\end{array}$} & \multicolumn{2}{|c|}{ Presencia VP } & \multicolumn{2}{|c|}{ Intensidad VP } & \multicolumn{2}{|c|}{ Mobbing } & \multicolumn{2}{|c|}{ Sintomas de Estrés } \\
\hline & $\begin{array}{l}\text { Alta - } \\
\text { Media }\end{array}$ & Nula & $\begin{array}{l}\text { Alta - } \\
\text { Media }\end{array}$ & $\begin{array}{l}\text { Baja - } \\
\text { Nula }\end{array}$ & $\begin{array}{l}\text { Alta - } \\
\text { Media }\end{array}$ & $\begin{array}{l}\text { Nula } \\
\text { / Baja }\end{array}$ & & Medio \\
\hline Cuarto nivel & 158 & 21 & 15 & 164 & 1 & 175 & 177 & 2 \\
\hline Tercer nivel & 5 & 3 & 0 & 8 & 0 & 8 & 8 & 0 \\
\hline -cuadrado & Pears & 03 & & 0,393 & & 0,669 & & 0,764 \\
\hline
\end{tabular}

VP=Violencia Psicológica.

ciones de docentes universitarios de otras investigaciones en cuanto se refiere a rangos de edad, nivel de formación, tiempos de dedicación. En el presente estudio no existe una asociación estadistica significativa entre las variables investigadas y los factores socio-demográficos laborales; similares resultados se obtuvieron en Herranza-Bellido, Reig-Ferrer y Cabrero Garcia ${ }^{1}$, quienes sostienen no haber encontrado diferencias estadísticamente significativas en mobbing debidas a la edad, género o categoría académica de los profesores. En México, Aldrete, Pando, Aranda y Torres ${ }^{14}$ realizan un estudio sobre Acoso Psicológico en el Trabajo, en donde las variables de edad y estado civil en las mujeres participantes no mostraron asociación significativa con ninguna de las diferentes mediciones del mobbing. En otro estudio realizado por Raya, Herruzo y Pino ${ }^{16}$ sobre los niveles de acoso psicológico en una población de 220 profesores (93 hombres y 127 mujeres) se evidenció que los factores socio-demográficos no guardan ninguna relación directa con la presencia de mobbing.

El presente estudio aporta con datos sobre la violencia psicológica en el trabajo e indica que un $87,2 \%$ de los investigados (163 docentes) manifiesta presencia de violencia psicológica, el 91,9 \% de los investigados (172 docentes) indica que la intensidad de la violencia psicológica es baja y nula y solamente 1 docente $(0,5 \%)$ indica que ha sido alta. Estos resultados tienen similitud con el estudio realizado por Cruz, Ovalle y Pando ${ }^{21}$, en académicos mexicanos, cuyos resultados indican que el 88,7\% (47 académicos) han sufrido de violencia psicológica; de acuerdo a la intensidad, el 95,7\% de ellos manifiesta que la violencia recibida es de baja intensidad y únicamente el 4,3\% de los académicos manifiesta que ha sido alta.

Pando ${ }^{17}$, en el estudio sobre Factores psicosociales y presencia de mobbing en profesores universitarios, indica que hasta un $82 \%$ del personal afirma haber recibido alguna forma de Violencia Psicológica en el Trabajo, porcentajes cercanos a los logrados en el presente estudio. Sobre el Acoso Psicológico (mobbing), 183 docentes $(97,8 \%)$ investigados indican que es nulo o bajo, mientras 4 docentes $(2,2 \%)$ indican que es medio y alto. En Colombia, el Ministerio de Trabajo en el 2004 llevó a cabo un estudio en que se reportó una prevalencia de acoso psicológico del 19,7\% para el sector salud y educación. En esta variable existe una diferencia significativa entre los dos estudios analizados; sin embargo, nuestros datos se acercan más a los resultados obtenidos por Pando ${ }^{17}$ en el estudio sobre Factores psicosociales y presencia de mobbing en profesores universitarios, en el cual 185 profesores respondieron a las encuestas y se encontró que 2 profesores -que representan el 1,08\% - respondieron que el nivel es alto, mientras 7 profesores -que representan el 3,74\% - indicaron que el nivel de acoso psicológico es medio, por otro lado hasta un $82 \%$ del personal afirma haber recibido alguna forma de Violencia Psicológica en el Trabajo.

En otro estudio realizado por Pando $^{27}$ (2012), los resultados indican que de la población investigada en el Ecuador (309 trabajadores) del sector industrial y de servicios, 273 trabajadores evaluados -que representan el 88,30\%- indican la presencia de violencia psicológica en el trabajo, 254 trabajadores -que representan el 82,1\%- indican que la intensidad es baja o nula, mientras que en referencia al Acoso Psicológico, 290 trabajadores -que representan el 93,9\%- indican que es bajo, mientras 3 trabajadores $(0,9 \%)$ indican que se encuentra en un nivel alto.

En referencia a los niveles de estrés, el presente estudio sostiene que 185 docentes $(98,9 \%)$ indicaron que el nivel es bajo, mientras 2 docentes $(1,1 \%)$ indicaron que es medio; los síntomas más frecuentes son: dolores de cuello o espalda, explosiones de furia, fatiga y gastritis.

En el estudio de Pozos, Horta, Delgadillo y Aguilera $^{22}$, los niveles de estrés crónico que se encontraron entre los odontólogos fueron: $11(12,2 \%)$ de la población total presentaron un nivel alto, $62(68,8 \%)$ presentaron un nivel medio y $17(18,9 \%)$ presentaron un nivel bajo. Las autoras sostienen que estos resultados concuerdan con otros estudios realizados donde se hace referencia a que, si bien el estrés afecta a ambos sexos, el impacto en cada uno es diferente. Con relación a la edad, se sabe que a mayor edad se va reduciendo la capacidad de su sistema inmune, mostrando significativamente más estrés y, si a ello le agregamos alteraciones en la estabilidad emocional por el estrés vivido a lo largo de varios años, las consecuencias pueden ser lamentables. La autora indica que: "La literatura consultada menciona que un nuevo estudio afirma que las personas de menos de 50 años con alta presión en su trabajo tienen un riego 70\% mayor de desarrollar enfermedades que aquellos que viven libres de estrés".

En el estudio realizado por Herranz ${ }^{1}$ se indica que el burnout, o estrés laboral asistencial, es un síndrome psicológico caracterizado por agotamiento emocional (CE), despersonalización (DP) e insatisfacción personal con los logros (RP). El estrés laboral asis- 
tencial se midió a través del Maslach Burnout lnventory (MBI) estableciéndose una situación definida por altas puntuaciones en CE y DE, y bajas en RP. Respondieron el cuestionario un total de 331 profesores y los resultados muestran que un reducido porcentaje de profesores, el 1,8\%, experimenta el síndrome de burnout o estrés laboral.

Un estudio que llama la atención es el realizado por Kinman ${ }^{31}$ realizado en Australia con 2609 académicos de 15 universidades, quienes reportan que el trabajo académico a tiempo completo es más estresante y que ha tenido un incremento de tareas: el $40 \%$ de académicos trabaja un exceso de 50 horas por semana; en relación con el perfil de daño, el 41\% de los docentes presenta sintomas de estrés; los trastornos del sueño, la ansiedad y la fatiga son manifestaciones de un tercio de los académicos participantes en esta investigación, sintomas que también predominan en los docentes ecuatorianos sujetos de la presente investigación.

\section{REFERENCIAS}

1. Herranz -Bellido J, Reig-Ferrer A, Cabrero-García J. La presencia de mobbing y sus determinantes laborales en profesores universitarios. Análisis y Modificación de conducta. 2006; 32(142):145-163.

2. Escartín-Solanelles J, Arrieta-Salas C, Rodriguez-Carballeira Á. Mobbing 0 acoso laboral: revisión de los principales aspectos teórico-metodológicos que dificultan su estudio. Actualidades en Psicología. 2009-2010;(23-24):1-19.

3. Aranda $C$, Pando M, Torres T, Salazar J, Sánchez JL. Factores psicosociales y patologías laborales en trabajadores de un sistema de transporte público urbano en México. Rev Col Psiquiatr. 2011; 40(2):266-278.

4. Einarsen $S$, Hoel, $H$, Zapf $D$, Cooper $C$. The Concept of Bullying at work. The European Tradition. In: Einarsen S, Hoel H, Zapf D, Cooper C, editors. Bullying and Emocional Abuse in the Workplace: Internacional Perspectives in research and practice. New York: Taylor \& Francis; 2003.

5. Leymann H. Mobbing. La persecution au travail. Paris: Du Seuil; 1996

6. Pando M, Ezqueda B. Temas de Condiciones de Trabajo y Salud Mental. Guadalajara: Universidad de Guadalajara; 2007.

7. González de Rivera J, Rodríguez-Abuín M. Cuestionario de estrategias de acoso psicológico: el LIPT-60 (Leymann Inventory of Psychological Terrorization) en versión española. Psiquis. 2003;24(2):59-69.

8. Peralta M. Manifestaciones del Acoso Laboral, mobbing y sintomas asociados al estrés postraumático: estudio de caso. Psicologia desde el Caribe [en línea] 2006 [citado 1 dic 2103]; 17:1-26. Disponible en: http://pepsic.bvsalud.org/ scielo.php?pid=S0123417X2006000100002\&script=sci_arttext

9. Riquelme A. Mobbing. Un tipo de violencia en el lugar de trabajo. Ciencias Sociales Online. 2006: 3(2):39-57.

10. González De Rivera J. Acoso Laboral Síntomas Clínicos; Escritores contra el Acoso Laboral [en línea] 2012 [citado el 29 mar 2013].Disponible en: www/ acosolaboral.net/gonzalez...joseluis-sintomas-clinicos-acoso-laboral.

11. Bustos E, Caputo M, Aranda S, Messoulam M. Psychological/Moral Workplace Harassment: Development of an Inventory in Argentina. Social Medicine. 2012; 6(4):241-245.

12. Martínez $E$, Agudelo $A$, Vásquez $E$. Mobbing, un aspecto a vigilar en los profesionales en Colombia. Rev Gerenc Polit Salud. 2010;9(19):41-527.

13. Herranz-Bellido J, Reig-Ferrer A, Cabrero-García J. La prevalencia del estrés laboral asistencial entre los profesores universitarios. Análisis y Modificación de Conducta. 2006; 32(146):743-766.

14. Aldrete $M$, Pando $M$, Aranda C,Torres T. Acosos Psicológicos en el Trabajo: ¿Un problema de género?. Enseñanza e Investigación en Psicología. 2006;11(1): 53-63.

15. Justicia F, Benítez J, Fernández E. Caracterización del acoso psicológico en el contexto universitario. Revista de Psicología del Trabajo y de las Organizaciones. 2006; 22(3):293-308.

16. Raya A, Herruzo J, Pino Ma. Acoso Psicológico (mobbing) en el ámbito educativo. Análisis y modificación de la conducta [en línea] 2007 [citado 29 dic 2012]; 33(144): 85-102. Disponible en: http:/rabida.uhu.es/dspce/bistream/ handle/10272/5816/Acoso_psicologico_mobbing_en_el_ambito_educativo. pdf?sequence $=2$.

17. Pando $\mathrm{M}$, Román $\mathrm{J}$, Acosta $\mathrm{M}$. Factores psicosociales de riesgo de trabajo en la empresa. La Habana: Red Iberoamericana de Riesgos Psicosociales Laborales; 2008.
18. Hiebert B, Farber I. Teacher Stress: A Literature Survey with a Few Surprises. Can J Educ.1984;9(1):14-27.

19. Matud M, Garcia M, Matud J. Estrés laboral y salud en el profesorado: un análisis diferencial del género y del tipo de enseñanza. Int J Clin Health Psychol. 2002;2(3):451-465.

20. León $R$, Avargues $L$. Evaluación del estrés laboral del personal universitario. MAPFRE Medicina. 2007;18(4): 323-332.

21. Cruz $P$, Ovalle $M$, Pando $M$. Mobbing y Estrés en Académicos de la Universidad de Ciencias y Artes de Chiapas. Memorias del 2do. Foro de las Américas en Investigación sobre Factores Psicosociales: Estrés y Salud en el trabajo. Guadalajara, Jalisco, 25-28 ago 2008. p.1-9.

22. Pozos R. Análisis comparativo de los niveles de estrés crónico de los Cirujanos Dentistas que laboran en las Instituciones de Salud Públicas. [Tesis de Doctorado en Psicología de la Salud]. Guadalajara: Universidad de Guadalajara; 2004.

23. Necsoi DV. Stress and Job satisfaction among University teachers. International Conference of Scientific Paper Afases; Brasov, Romania 26-28 may 2011. Brasov: University of Brasov; 2011.

24. Pando M, Aranda C, Preciado L, Franco A, Salazar J. Validez y confiabilidad del inventario y acoso psicológico en el trabajo (IVAPT-PANDO). Enseñanza e Investigación en Psicología. 2006;11(2):319-332.

25. Zapata L, Restrepo C. Propuesta de los elementos a tener en cuenta en la prevención primaria del mobbing o acoso laboral en el contexto organizacional. Medellin: Universidad de Buenaventura; 2009.

26. Macías S. Aplicación y evaluación psicométrica del test IVAPT-PANDO en dos centros de atención primaria. Psicología y Salud. 2008;18(2): 247-254.

27. Pando $M$, Aranda $C$, Olivares $D$. Análisis factorial confirmatorio del inventario de violencia y acoso psicológico en el trabajo (IVAPT-PANDO) para Bolivia y Ecuador. Liber. 2012;18(1):27-36.

28. Aldrete $\mathrm{M}, \mathrm{Cruz}$ O. Estrés Laboral y Burnout en Docentes de Educación Superior en México. México: UNICACH; 2013.p 82. (Colección Montebello).

29. Domínguez T, Méndez M, Meza B. Importancia de la elaboración de instrumentos en el estudio del estrés. 1er. Coloquio Interno de Investigación: Estrés y Salud. Evaluación y Procedimientos No- Invasivos para su manejo en poblaciones de alto riesgo. México: UNAM; 1994.

30. Morales A. Activación, ansiedad y sintomas de estrés en una muestra de trabajadores. Universidad Autónoma de San Luis Potosí. Revista científica electrónica de psicología: 2008; 5

31. Kinman G. Pressure Points: A review of research on stressors and strains in UK academics. Educ Psychol-UK. 2001; 21(4):273-492.

\section{Referencias complementarias:}

Carrión G, López B, Pando M. Inventario de Violencia y Acoso Psicológico en el Trabajo. IVAPT-E-R. Barcelona: AEPA; 2007.

Pozos R, Torres L, Aguilera V, Acosta F, González P. Stress associated Factors in Mexican Dentists. Braz Oral Res. 2008; 22(3):223-228. 